\title{
ELEMENTOS DA TEORIA DAS FILAS
} OSWALDO FADIGAS TORRES

\begin{abstract}
"As teorias podem ser descritas em têrmos simples. ainda que se refiram a fenômenos que não pareçam sê-lo." - J. D. WIILIAMs
\end{abstract}

Com êste artigo, exposição simplificada da teoria das filas, pretendemos vir de encontro à necessidade de muitos administradores que, desejosos de estudar o assunto, difícilmente encontram trabalhos em nossa língua versando de maneira acessivel êsse importante tópico.

Datando embora a teoria e suas aplicações de meio século, sua utilização tem sido ainda restrita no Brasil. Porém, com o desenvolvimento industrial, o crescimento das emprêsas e a necessidade de racionalização que ora se verifica, pode-se prever que, nos anos imediatos, veremos nossos administradores recorrerem com insistência aos conceitos e métodos da teoria das filas de espera nos setores de fabricação, manutenção, transporte, tráfego, comunicações, vendas e serviços em geral.

A teoria das filas de espera é um método estatístico que permite estimar as demoras que ocorrem quando um serviço tem de ser proporcionado a clientes cuja chegada se dê ao acaso, como, por exemplo, fregueses que esperem

Oswaldo Fadigas Tôrres - Professor de Tempo Parcial, do Departamento de Métodos Quantitativos, da Escola de Administnação de Emprêsas de São Paulo, da Fundação Getúlio Vargas. Livre-Docente e Professor-Assistente de Planejamento da Produção da Escola Politécnica da Universidadoi de S. Paula, 
para ser atendidos numa loja e automóveis que se congestionem num pôsto de pedágio.

Um dos primeiros trabalhos efetuados sôbre o tema foi o de A. K. ERLANG, em relação a circuitos telefônicos, para a Companhia Telefônica de Copenhagen, em 1908. A Segunda Grande Guerra deu forte impulso a aplicações militares, verificando-se, terminada a conflagração, inúmeras aplicações da teoria na área civil.

\section{CONCEITO E CLASSIFICAÇ̃̃o DAS FILAS}

Em sua forma mais familiar uma fila é gerada quando unidades (clientes), chegando a um pôsto de serviço, não possam ser atendidas prontamente, tendo, ocasionalmente, que esperar para sê-lo. O grupo que espera é a fila; porém, êsse têrmo geralmente indica todos os clientes presentes, isto é, os que esperam e os que estejam sendo atendidos.

A estrutura básica do problema é bastante geral, de modo que muitas situações práticas, que aparentemente não constituem filas, podem ser estudadas através da teoria das filas.

Normalmente, a fila resulta da falta - deliberada ou não - de programação, pois, se fôsse possível organizar as chegadas e os serviços, seria também possível evitar completamente a espera dos clientes e não haveria fila. $\mathrm{Na}$ maioria dos casos, porém, é impossível programar; assim, as filas, embora não desejadas, são inevitáveis.

O processo de fila é caracterizado por três elementos:

1. Regime de chegada;

2. Regime de serviço; e

3. Disciplina da fila.

O regime de chegada inclui os seguintes elementos:

1. Especificação da população de clientes; finita ou infinita; . 
2. Distribuição da probabilidade do intervalo de $t \in m p o$ entre chegadas: essa distribuição pode ser estacionária ou variável no tempo, pode depender do tamanho da fila etc..

No regime de serviço existem três aspectos a ser considerados:

1. A disponibilidade do serviço: alguns sistemas só atendem durante um certo intervalo de tempo; outros estão sempre disponíveis;

2. A capacidade do sistema, isto é, o número de clientes atendidos simultâneamente;

3. A duração do tempo de serviço de cada cliente, que pode ser constante ou aleatória, com distribuição de probabilidade estacionária ou não, dependendo, inclusive, do tamanho da fila.

A disciplina da fila é o conjunto de regras que determinam a ordem em que os clientes são atendidos. Há várias possibilidades: atendimento pela ordem de chegada, atendimento aleatório, prioridade para certas categorias de clientes etc..

\section{SISTEMAS ERGÓDICOS}

A fila é um processo estocástico, isto é, seu estado num instante $t$ (número de clientes presentes, tamanho da fila, tempo de espera etc.) é variável aleatória. $O$ estado do sistema é, realmente, um evento condicionado, cuja probabilidade no instante $t$ depende do valor da probabilidade no instante inicial $t$.

Em muitos casos, essa dependência do estado inicial desaparece depois de certo tempo (mais precisamente quando $t \rightarrow \infty$ ) isto é, o sistema atinge posição de equilíbrio (regime permanente), em que as probabilidades não dependem mais do estado inicial, nem do tempo decorrido. 
Se a convergência ao limite é rápida, então, torna-se possível usar essa solução-limite como aproximação para calcular a probabilidade dos diversos estados. Os sistemas que gozam dessa propriedade de as probabilidades tenderem para uma situação de equilíbrio são denominados sistemas ergódicos.

\section{CRITÉRIOS DE AVALIAÇÃo}

Dependendo das circunstâncias, podemos avaliar o funcionamento de um sistema por diversos critérios:

1. Probabilidade de espera $P(D)=P(T>0)$. Quando o custo de uma demora no atendimento é grande, a escolha de um valor pequeno para $P(D)$ pode ser o critério adequado. Exemplo: problemas de estoque.

2. Probabilidade de espera maior que $t, P(T>t)$. Êsse é o critério adequado se os clientes não toleram a espera por mais do que certo tempo. Exemplo: aviões esperando para aterrissar.

3. Tempo médio de espera $E[T]$. Êsse interessa quando o conjunto de demoras - e não uma demora individual - é importante. Exemplo: tempo perdido numa fábrica por máquinas paradas aguardando serviço.

4. Probabilidade de a fila ser maior do que um certo valor $m: P(M>m)$. É importante quando devemos determinar a dimensão do espaço para acomodar a fila. Exemplo: número de cadeiras na sala de espera de um consultório médico.

5. Tamanho médio da fila $E[M]$. Como o tempo total gasto em espera por todos os clientes em conjunto é igual ao produto do número médio de clientes na fila pelo tempo em que a fila existe, segue-se que o tamanho médio da fila dá também a perda de tempo por unidade de tempo e, portanto, pode ser usado para avaliar o tempo total perdido na fila. Exemplo: tem- 
po total perdido por empregados esperando na fila para serem atendidos no almoxarifado da emprêsa.

6. Perda de tempo relativa. Em muitos casos, o critério de avaliação é a perda de tempo relativa, definida como a relação entre o tempo de espera e o tempo de serviço.

ESTUDO DE UM CASO SIMPLES

A maior parte das situações de fila exige desenvolvimento matemático difícil, sendo preferível, nesse caso, usar métodos de simulação, que discutiremos adiante. Para dar ao leitor idéia do tratamento matemático, analisaremos sistema bastante simples, com chegadas segundo uma distribuição de Poisson de média $\lambda t$, um único pôsto de serviço com distribuição exponencial dos tempos de atendimento de média $1 / \mu$ e disciplina de atendimento pela ordem de chegada.

Sabemos que o que caracteriza as distribuições de Poisson e a exponencial é o fato de, dado um intervalo de tempo $\Delta t$ suficientemente pequeno:

1. A probabilidade de uma chegada (ou de uma saída por término de serviço) ser proporcional a $\Delta t$, isto é, será respectivamente $\lambda \Delta t$ ou $\mu \Delta t$.

2. A probabilidade de mais de uma chegada ou saida em $\Delta t$ ser desprezível. Em conseqüência, num intervalo $\Delta t$ só podemos ter uma chegada, com possibilidade $\lambda \Delta t$, ou nenhuma, com probabilidade $1-\lambda \Delta t$. O mesmo vale para os términos de serviço em $\Delta t$ : ou termina um serviço, com probabilidade $\mu \Delta t$, ou então nenhum, com probabilidade $1-\mu \Delta t$.

Nessas condições, a probabilidade de que existam $n>0$ clientes no sistema, no instante $t+\Delta t$, pode ser expressa pela soma das probabilidades dos seguintes eventos: 
a) existem $(n-1)$ clientes no instante $t$, há uma chegada no intervalo $\Delta t$ e não há término de. serviço em $\Delta t$;

b) existem $n$ clientes no instante $t$ e não há nem chegada nem saída no intervalo $\Delta t$;

c) existem $n$ clientes no instante $t$, há uma chegada e uma saída no intervalo $\Delta t$;

d) existem $(n+1)$ clientes no instante $t$, não há chegada e há uma saída no intervalo ${ }^{\Delta} t$.

Para o caso $n=0$, basta considerar as duas situações seguintes:

a) existem 0 clientes no instante $t$ e não há chegada no intervalo $\Delta t$;

b) existe 1 cliente no instante $\Delta t$, não há chegada, e há uma saída no intervalo $\Delta t$.

Temos, portanto, indicando por $\boldsymbol{P}_{n}(t)$ a probabilidade de haver $n$ clientes no sistema no instante $t$ :

$$
\begin{array}{ll}
P_{0}(t+\Delta t)=P_{0}(t)(1-\lambda \Delta t)+P_{1}(t) \cdot \mu \Delta t \\
P_{n}(t+\Delta t)=P_{n-1}(t) \cdot \lambda \Delta t \cdot(1-\mu \Delta t)+ \\
+P_{n}(t)(1-\lambda \Delta t)(1-\mu \Delta t)+P_{n}(t) \cdot \lambda \Delta t \cdot \mu \Delta t+ \\
+P_{n+1}(t)(1-\lambda \Delta t) \cdot \mu \Delta t & n>0
\end{array}
$$

Calculando a expressão $\frac{P_{n}(t+\Delta t)-P_{n}(t)}{\Delta t}$ e fazendo $\Delta t$ tender a zero, lembrando que, no equilíbrio, as probabilidades não dependem de $t$ e, portanto, a derivada $\frac{\mathrm{dP}_{\mathrm{n}}(\mathrm{t})}{\mathrm{dt}}$ é nula, temos, indicando por $\boldsymbol{P}_{n}$ a probabilidade de equilíbrio de haver $n$ clientes no sistema: 


$$
\begin{aligned}
& \frac{\mathrm{dP}(\mathrm{t})}{\mathrm{dt}}=-\lambda \mathrm{P}_{\mathrm{o}}+{ }^{\mu} \mathrm{P}_{1}=0 \\
& \frac{\mathrm{dP} \mathrm{P}_{\mathrm{n}}(\mathrm{t})}{\mathrm{dt}}=\lambda \mathrm{P}_{\mathrm{n}-1}-(\lambda+\mu) \mathrm{P}_{\mathrm{n}}+\mu \mathrm{P}_{\mathrm{n}_{+1}}=0 \quad \mathrm{n}>0
\end{aligned}
$$

Resolvendo por substituição sucessiva, temos:

$$
\begin{aligned}
& \mathbf{P}_{1}=\frac{\therefore}{\mu} \mathbf{P}_{0} \\
& \mathbf{P}_{2}=\left(\frac{\lambda}{\mu}\right)^{2} \mathbf{P}_{0} \\
& \mathbf{P}_{\mathrm{n}}=\left(\frac{\lambda}{\mu}\right)^{n} \mathbf{P}_{0}
\end{aligned}
$$

Para obter $\mathbf{P}_{0}$ lançamos mão da condição:

$$
\begin{aligned}
& \underset{n=0}{\sum_{n} P_{n}}=1 \text {, e temos: } \\
& P_{0}\left[1+\left(\frac{\lambda}{\mu}\right)+\left(\frac{\lambda}{\mu}\right)^{2}+\ldots+\left(\frac{\lambda}{\mu}\right)^{n}+\ldots\right]=1 .
\end{aligned}
$$

A expressão entre colchêtes é a soma de uma progressão geométrica de razão $\lambda / \mu$, com infinitos têrmos. Para que a soma seja convergente devemos ter $\frac{\lambda}{\mu}<1$, o que é evidente por considerações de ordem física, pois sendo $\lambda$ o número médio de chegadas na unidade de tempo e sendo ${ }^{\mu}$ o número médio de saídas (quando haja clientes no sistema) se $\lambda>\mu$, então haveria congestionamento crescente.

Em virtude da importância da relação $\left(\frac{\lambda}{\mu}\right)$ ela recebe nome e símbolo especiais: chama-se fator de utilização, ou intensidade de tráfego. Ela tem uma unidade própria: 
é medida em "erlangs" (em homenagem a ERLANG, pioneiro da teoria das filas). Utilizaremos a letra grega $\rho$ para indicar o fator de utilização, isto é:

$$
\rho=\frac{\lambda}{\mu}
$$

Voltando à determinação de $P_{o}$, se $\rho=\frac{\lambda}{\mu}<1$ temos, então:

$$
\left[1+\rho+\rho^{2}+\ldots \ldots+\rho^{\mathrm{n}}+\ldots\right]=\frac{1}{1-\rho}(\rho<1)
$$

e as probabilidades em regime permanente são:

$$
\begin{aligned}
& \mathbf{P}_{o}=(1-\rho) \\
& \mathbf{P}_{\mathbf{n}}=(1-\rho) \rho^{n}
\end{aligned}
$$

Temos, também:

$\operatorname{Prob}[\mathrm{N}>\mathrm{n}]=\sum_{\mathrm{n}_{+1}}^{\infty} \mathbf{P}_{\mathrm{n}}=(1-\rho)\left[\rho^{\mathrm{n}+1}+\rho^{\mathrm{n}+2}+\ldots\right]=$

$=(1-\rho) \rho^{\mathrm{n}+1}\left[1+\rho+\rho^{2}+\ldots\right]=\rho^{\mathrm{n}+1}$

A probabilidade em particular de o sistema estar ocupado será:

$$
P(N>0)=P,
$$

o que justifica a denominação "fator de utilização".

O sistema estará vazio durante percentagem do tempo igual a $P_{o}$, isto é $(1-\rho)$.

Conhecida a distribuição de probabilidade de $\mathrm{N}$, podemos calcular o número médio de clientes presentes no sistema:

$$
\begin{aligned}
& \mathrm{E}[\mathrm{N}]=\sum_{0}^{\infty} \mathrm{nP} \mathrm{P}_{\mathrm{n}}=(1-\rho)\left[\rho+2 \rho^{2}+3 \rho^{3}+\ldots+\mathrm{n} \rho^{\mathrm{n}}\right]= \\
& =\rho(1-\rho)\left[1+2 \rho+3 \rho^{2}+\ldots+\mathrm{n} \rho^{\mathrm{n}-1}+\ldots\right]
\end{aligned}
$$


A expressão entre colchêtes é fàcilmente calculada observando que é a derivada de $\left[1+\rho+\rho^{2}+\ldots+\rho^{\mathrm{n}}\right]$ e portanto vale $\frac{1}{(1-p)^{2}}$.

Logo: o número médio de clientes no sistema é:

$$
\mathrm{E}(\mathrm{N})=\frac{\rho}{1-\rho}=\frac{\lambda}{\mu-\lambda}
$$

Para determinar o comprimento médio da fila, basta notar que se $M$ é o número de clientes na fila, temos:

$$
\begin{aligned}
& \mathbf{M}=0 \text { se } \mathbf{N}=0 \\
& \mathbf{M}=0 \text { se } \mathbf{N}=1 \\
& \mathbf{M}=\mathbf{N}-1 \text { se } \mathbf{N}>1
\end{aligned}
$$

Portanto, a distribuição de probabilidade de $\mathbf{M}$ é:

$$
\begin{aligned}
& \mathbf{P}(\mathbf{M}=0)=(1-\rho)+(1-\rho) \rho=1-\rho^{2} \\
& \mathbf{P}(\mathbf{M}=\mathbf{m})=(1-\rho) \rho^{\mathrm{m}+1} \quad(\mathrm{~m} \geqslant 1)
\end{aligned}
$$

O tamanho médio da fila é:

$$
\begin{gathered}
\mathrm{E}[\mathrm{M}]=\stackrel{\sum}{1}_{=}^{\infty} \mathrm{m}(1-\rho) \rho^{\mathrm{m}+1}= \\
=(1-\rho) \rho^{2}\left[1+2 \rho+3 \rho^{2}+\ldots\right]= \\
\mathrm{E}[\mathrm{M}]=\frac{\rho^{2}}{1-\rho}=\frac{\lambda^{2}}{\mu(\mu-\lambda)}
\end{gathered}
$$

Êsse valor inclui as filas de tamanho zero. Em geral, interessa-nos o tamanho médio da fila, quando há fila, isto é, $\mathrm{E}(\mathbf{M} \mid \mathbf{M}>0)$. Como $\mathbf{P}(\mathbf{M}=0)=1-\rho^{2}$, segue-se que 
$P(M>0)=\rho^{2}$ e, portanto, o tamanho médio da fila (quando há fila) é:

$$
\mathrm{E}(\mathrm{M} \mid \mathrm{M}>0)=\frac{1}{1-\rho}=\frac{\mu}{\lambda-\mu}
$$

Distribuição dos Tempos

O tempo total $\mathrm{V}$ gasto por um cliente no sistema é a soma do tempo de espera $\mathrm{W}$ e do tempo de serviço $\mathrm{S}$, isto é: $\mathrm{V}=\mathrm{W}+\mathrm{S}$.

Vejamos, primeiramente, como se distribui o tempo de espera W. A probabilidade de um cliente não ter de esperar $(W=0)$ é a de encontrar o sistema vazio, isto é, $\mathrm{P}(\mathrm{W}=0)=\mathrm{P}_{0}=1-\rho$. Isso quer dizer que $\mathrm{W}$ tem uma distribuição de probabilidade mista, pois é discreta (no ponto $\mathrm{W}=0$ ) e tem uma parte contínua (região $W>0$ ), com densidade de probabilidade $f(W)$.

Para determinar $f(W)$ observamos que o evento (espera de um cliente estar situado entre $w$ e $w+d w$ ) é a composição, para $\mathrm{n}=1$ até $\mathrm{n}=\infty$ dos eventos: (n elementos no sistema antes dêsse cliente chegar); $[(n-1)$ serviços no intervalo $\mathrm{w}$ ]; ( 1 serviço $\mathrm{em} \mathrm{dw}$ ).

Temos, portanto:

$$
\begin{aligned}
& \mathrm{P}[\mathrm{w} \leq \mathrm{W} \leq \mathrm{w}+\mathrm{dw}]= \\
& \sum_{\mathrm{n}=1}^{\infty}\left[(1-\rho) \rho^{\mathrm{n}}\right]\left[\frac{\left({ }^{\mu} \mathbf{w}\right)^{\mathrm{n}-1} \mathrm{e}^{-\mu^{\mathrm{w}}}}{(\mathrm{n}-1) !}\right]\left[{ }^{\mu} \mathrm{dw}\right]= \\
& \quad=\mathbf{f}(\mathbf{w}) \mathrm{dw} .
\end{aligned}
$$

Simplificando, temos:

$$
f(w)=(1-\rho) e^{-\mu^{w} \sum_{n=1}^{\infty} \frac{\lambda^{n} w^{n-1}}{(n-1) !}}=\left(1-\frac{\lambda}{\mu}\right) \lambda e^{-\left(\mu-\lambda^{\prime} w\right.}
$$




$$
f(w)=\frac{\lambda}{\mu}(\mu-\lambda) e^{-(\mu-\lambda) w}
$$

Evidentemente $\int_{0}^{\infty} f(w) d w=\frac{\lambda}{\mu} \int_{0}^{\infty}(\mu-\lambda) e^{-(\mu-\lambda)^{w}} d w=$ $=\frac{\lambda}{\mu}=\rho$, pois temos uma probabilidade $\mathrm{P}(\mathrm{W}=0)=1-\rho$.

A distribuição dos tempos de espera condicionada a haver espera será:

$$
f(w / W>0)=\frac{f(w)}{P(w>0)}=\frac{f(w)}{\rho}=\left(\mu_{-\lambda}\right) e^{-\left(\mu_{-\lambda}\right) w}
$$

E, portanto, uma exponencial de parâmetro $(\mu-\lambda)$ e podemos escrever diretamente:

Tempo médio de espera (quando há espera)

$$
\mathrm{E}(\mathrm{W} / \mathrm{W}>0)=\frac{1}{\mu-\lambda}
$$

Evidentemente, teremos:

Tempo médio de espera (incluindo o zero)

$$
\mathrm{E}(\mathrm{W})=\frac{\lambda}{\mu(\mu-\lambda)}
$$

Para determinar a distribuição de probabilidade do tempo total $\mathrm{V}$ gasto no sistema por um cliente, basta lembrar que um segundo cliente, que chegue imediatamente depois 
dêsse, irá demorar um tempo $\mathrm{W}=\mathrm{V}$ para ser atendido; logo, a distribuição de $\mathrm{V}$ é a mesma de (W/W>0), isto é:

$$
g(v)=(\mu-\lambda) e^{-(\mu-\lambda) v}
$$

Tempo médio gasto no sistema

$$
\mathrm{E}(\mathrm{V})=\frac{1}{\mu-\lambda}
$$

Probabilidade de um cliente ter de esperar mais que um tempo $v$ :

$$
\mathrm{P}(\mathrm{V}>\mathrm{v})=\mathrm{e}^{-\left(\mu-\lambda^{) \mathrm{v}}\right.}
$$

Determinaremos a seguir os tempos médios de espera e de atendimento por outro método, utilizando a idéia de fluxo médio no regime permanente.

Em média, chegam no sistema $\lambda$ clientes por unidade de tempo; evidentemente, estando em equilíbrio, saem em média $\lambda={ }^{\mu} \rho$, isto é, são atendidas em média ${ }^{\mu}$ pessoas durante um intervalo $\rho$ (fração de tempo em que o sistema está ocupado).

Como há em média $E(N)=\frac{\rho}{1-\rho}=\frac{\lambda}{\mu-\lambda}$ clientes no sistema, a demora média no sistema é:

$$
E(V)=\frac{E(N)}{\lambda}=\frac{1}{\mu-\lambda}
$$

O tempo médio na fila será $\mathrm{E}[\mathrm{W}]=\frac{\mathbf{E}(\mathrm{M})}{\lambda}=\frac{\lambda}{\mu(\mu-\lambda)}$. 
Êsse resultado também poderia ser obtido de $\mathrm{E}[\mathrm{V}]=$

$=\mathrm{E}[\mathrm{W}]+\mathrm{E}[\mathrm{S}]$ ou $\mathrm{E}[\mathrm{W}]=\mathrm{E}[\mathrm{V}]-\mathrm{E}[\mathrm{S}]=$

$=\frac{1}{\mu-\lambda}-\frac{1}{\mu}=\frac{\lambda}{\mu(\mu-\lambda)}$.

\section{Exemplo de Aplicação}

Um almoxarifado recebe em média 12 pedidos por hora, com distribuição de Poisson. O encarregado pode atender em média 20 pedidos por hora, tendo o tempo de atendimento distribuição exponencial. Calcular o tempo médio perdido na fila, o tempo médio gasto na fila por um operário e a porcentagem de tempo em que o encarregado não tem o que fazer.

Temos: $\lambda=12 /$ hora

$$
\mu=20 / \text { hora } \quad \rho=
$$

$=\frac{\lambda}{{ }^{\mu}}=0,60$.

Probabilidade de estar desocupado P. $=1-\rho=0,40$. Tamanho médio da fila, quando há fila:

$$
\mathrm{E}[\mathrm{M} \mid \mathrm{M}>0]=\frac{1}{1-\rho}=2,5 \text { homens. }
$$

Isso equivale a dizer que, em cada hora de trabalho da fábrica, há uma perda de tempo de 2,5 homens-horas na fila.

O tempo médio de espera é:

$\mathrm{E}[\mathrm{W}]=\frac{\lambda}{\mu(\mu-\lambda)}=\frac{12}{20(20-12)}=0,075 \mathrm{~h}=$ $=4,5$ minutos.

O tempo médio de espera quando há fila é:

$\mathrm{E}[\mathrm{W} \mid \mathrm{W}>0]=\frac{1}{\mu-\lambda}=\frac{1}{8}=0,125 \mathrm{~h}=7,5$ minutos. 
Êsse é também o tempo médio gasto no sistema.

$\mathrm{E}[\mathrm{V}]=0,125 \mathrm{~h}=7,5$ minutos.

SOLUÇÃO POR SIMULAÇÃO

Em casos complexos o estudo do comportamento do sistema pode ser feito por simulação. Para tal, devemos gerar aleatòriamente chegadas e serviços, de modo a reproduzir o funcionamento do sistema durante um período de tempo suficientemente longo para que as médias calculadas sejam representativas do sistema em equilíbrio.

Para gerar eventos de uma distribuição aleatória utilizamos a curva das probabilidades ou frequiências acumuladas que, como sabemos, vai de zero a um. Sorteamos um número ao acaso (tirado de uma tabela de números eqüiprováveis, por exemplo) e o transformamos em porcentagem, de modo a ter sempre números entre zero e um. O valor da variável aleatória cuja probabilidade acumulada corresponder ao número sorteado será o valor escolhido para a simulação.

Para exemplificar, simularemos o funcionamento do sistema utilizado anteriormente como exemplo.

Obedecendo as chegadas a uma Poisson de média 12/hora ou 0,20 /minuto (Gráfico 1 ), sabemos que o intervalo entre duas chegadas consecutivas será uma exponencial de média $\frac{1}{0,20}=5$ minutos (Gráfico 2 ) cuja curva de probabilidades acumuladas tem a expressão: $F(t)=$ $=1-\mathrm{e}^{-0,20 t}$.

Para gerar intervalos de tempo desta distribuição podemos utilizar um gráfico ou uma tabela.

Assim, por exemplo, se o número sorteado na tabela de números equiiprováveis foi 768 , procuramos na tabela o valor de $t$ mais próximo, por excesso, de 0,768 , e encontramos $\mathrm{t}=8$ minutos, valor que utilizaremos como intervalo para a próxima chegada. 

GRÁFICO 1: As chegadas obedecem
a uma distribuição de Poisson

GRÁFICO 2: O intervalo entre duas chegadas consecutivas obedece a uma

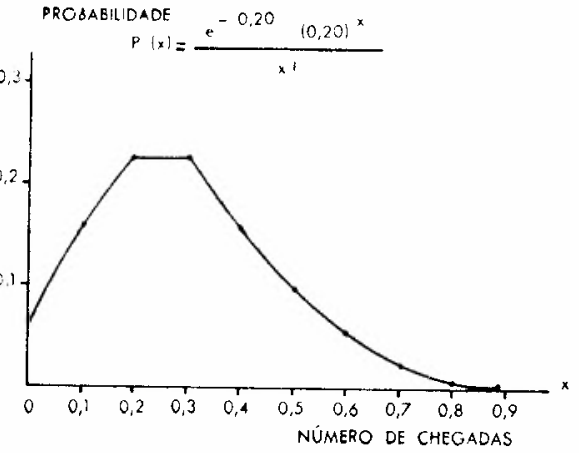
distribuição exponencial negativa

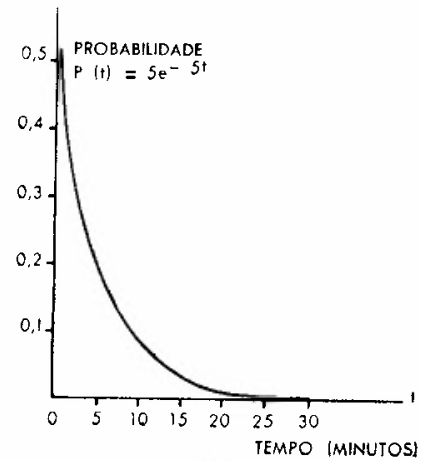

De maneira idêntica simularemos os tempos de serviço, a partir da distribuição exponencial de média 3 minutos ( 20 atendimentos por hora), usando a tabela de $F(s)=$ $=1-\mathrm{e}^{-\mathrm{t} / 3}$.

TABELA DE $1-e^{-t / 5}$

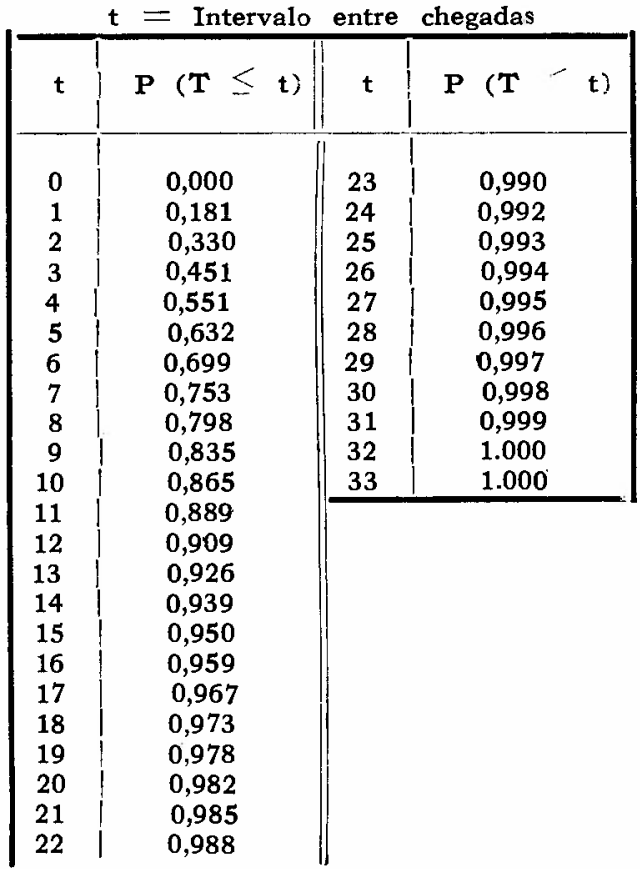

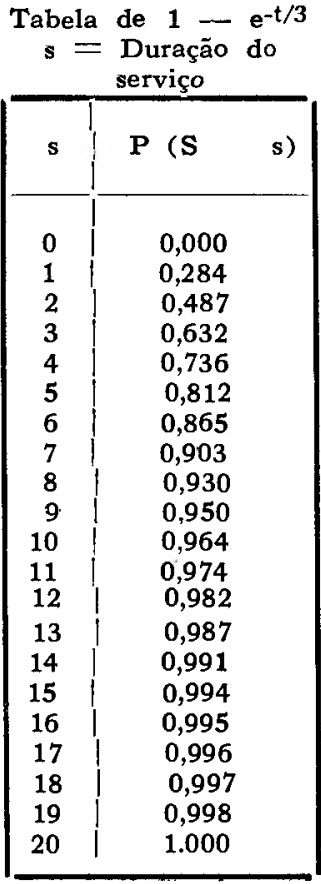




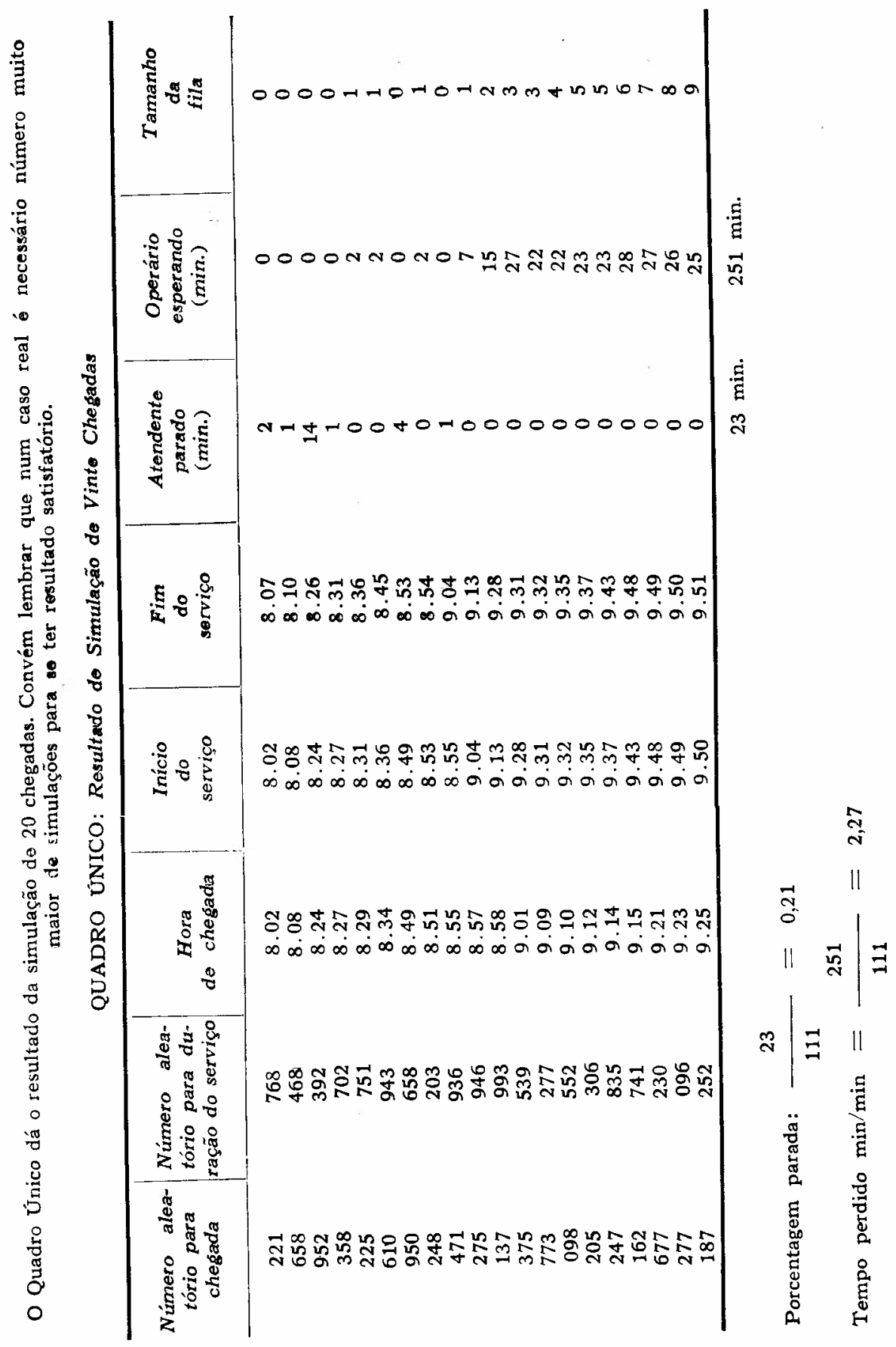




\section{CONCLUSÃO}

Em contraste com a extensa literatura estrangeira existente sôbre a teoria das filas, pouco se tem escrito em nosso País sôbre o assunto. A administração de emprêsas está evoluindo rápidamente para fase de crescente utilização da metodologia quantitativa. O administrador enfrenta o desafio de ter que utilizar métodos especiais, que pressupõem conhecimentos matemáticos extensos $e$, às vêzes, altamente avançados, sem possuir êsses conhecimentos de base. Aos leitores ansiosos por se atualizar nas áreas da Pesquisa Operacional expusemos, neste artigo limitando nossa apresentação aos casos mais simples - o essencial sôbre a teoria e a aplicação das filas de espera.

\section{BIBLIOGRAFIA}

D. R. Cox e W. L. Smith, Queues, Nova Iorque: Wiley \& Sons, 1961.

P. M. Morse, Queues, Inventories and Maintenance, Nova Iorque: Wiley \& Sons, 1958.

M. Sasieni, A. Yaspan e I. Friedman, Operations Research, Nova Iorque: Wiley \& Sons, 1959.

T. I. SAATY, Elements of Queueing Theory, Nova Iorque: McGraw-Hill Book Co., 1961. 